Images du travail, travail des images

\title{
Ce que la caméra peut faire (dire) aux techniques : la médiation cinématographique et le destinataire (trouble) du geste
}

The camera's impact on technical sayings and doings: cinematographic mediation and "blurry recipients" of gestures

\section{Baptiste Buob}

\section{OpenEdition \\ Journals}

Édition électronique

URL : http://journals.openedition.org/itti/1055

DOI : 10.4000/itti. 1055

Éditeur

Université de Poitiers

Référence électronique

Baptiste Buob, «Ce que la caméra peut faire (dire) aux techniques : la médiation cinématographique et le destinataire (trouble) du geste », Images du travail, travail des images [En ligne], 3 | 2017, mis en ligne le 01 février 2017, consulté le 14 avril 2021. URL : http://journals.openedition.org/itti/1055 ; DOI : https://doi.org/10.4000/itti.1055

Ce document a été généré automatiquement le 14 avril 2021

Images du travail, travail des images 


\section{Ce que la caméra peut faire (dire) aux techniques : la médiation cinématographique et le destinataire (trouble) du geste}

The camera's impact on technical sayings and doings: cinematographic mediation and "blurry recipients" of gestures

Baptiste Buob

\section{NOTE DE L'ÉDITEUR}

Une version plus courte de ce texte est à paraître dans l'ouvrage Gestes techniques, techniques du geste édité par D. Bouillon, A. Guillerme, M. Mille et G. Piernas (Lille, Presses universitaires du Septentrion).

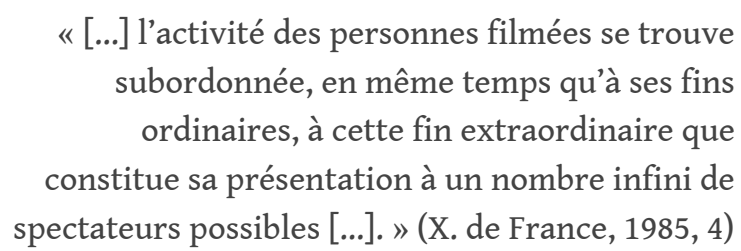

Au terme de la projection du film Luthiers de la main à la main traitant des relations de transmission et de leurs évolutions dans le domaine de la lutherie française (Buob, 2013a), un des protagonistes est venu m'exprimer sa surprise après avoir vu ses propres gestes projetés sur l'écran : «Normalement je ne travaille pas comme ça!» Le luthier constatait de lui-même que la présence de la caméra l'avait incité à modifier ses gestes « habituels $»^{1}$. 
Ce média ne peut être affiché ici. Veuillez vous reporter à l'édition en ligne http://

Cette anecdote appelle des interrogations qui reviennent inlassablement aux oreilles des anthropologues-cinéastes : quels sont les effets dus à la présence d'une caméra sur une situation et, s'il y en a, ne modifient-ils pas les informations obtenues au point de les rendre inutilisables? Bien que s'inscrivant dans une longue histoire de réflexions sur le statut des images et les conditions de leur obtention, le débat concernant les supposés effets provoqués par les instruments de prises de vues n'a pas cessé de faire couler de l'encre : la question des "perturbations » dues à la présence d'une caméra interroge toujours les chercheurs en sciences humaines.

Dans le domaine des réflexions sur les procédures et les formes de la représentation scientifique, continue à exister, sous une forme à peine renouvelée, la controverse qui opposait au début du XXe siècle les deux vertus épistémiques de l'objectivité mécanique et de la subjectivité artistique (Daston et Galison, 2012). Tenants d'une objectivité mécanique, les chercheurs en sciences du comportement mettent en place des dispositifs de captation extrêmement contrôlés visant à minimiser les modifications des actions filmées en réduisant au maximum les perturbations. Dans le prolongement des travaux des pionniers du cinéma scientifique, le film est alors conçu comme un instrument d'enregistrement au service d'une fine analyse des mouvements corporels (Bril, 2012). En revanche, dans la perspective du film ethnographique, qui selon une définition aux contours d'oxymore vise à associer « la rigueur de l'enquête scientifique à l'art de l'exposé cinématographique » (Rouch, 1968, 433), la caméra n'est plus conçue comme un strict instrument de mesure. Suivant les principes de tournage proches de ceux du " cinéma direct ", le cinéaste revendique alors une transformation du réel afin non seulement d'en creuser la densité, mais aussi de restituer au spectateur une part de sa profondeur dans une forme explicite et attractive ${ }^{2}$. Aussi les images animées deviennent « film » ethnographique lorsqu'elles ont d'autres prétentions que celle de constituer des notes audiovisuelles qui pourront être décortiquées et analysées en vue de publications écrites ultérieures. Mise en forme diégétique d'une recherche, le film ethnographique n'est pas non plus une «simple» mise en image de recherches antérieures mais se situe à l'interface des deux «tendances-cadres» que sont l'exploration et l'exposition ( $\mathrm{Cl}$. de France, 1989 [1982], XV). L'ethnologue-cinéaste transforme le visible en reliant certains de ces éléments, en variant les angles et les cadrages, en incluant ou en excluant, en soulignant et en estompant, etc. De ce point de vue, ce que dit François Laplantine à propos de la photographie est également valable pour le film : il fait «advenir des formes qui n'existaient pas avant» $(2007,50)$. Et le film doit dès lors être conçu comme un de ces "médiateurs " qui «transforment, traduisent, distordent, et modifient le sens ou les éléments qu'ils sont censés transporter. » (Latour, 2006, 58)

5 Selon cette optique, laquelle correspond le plus à mon approche et au genre de cinéma dont il sera ici question, il faut admettre que s'il peut bien y avoir «perturbation », la notion doit alors être envisagée selon l'acception proposée par Georges Devereux et considérée comme une précieuse source de connaissance ${ }^{3}$ :

«Au lieu de déplorer la perturbation due à notre présence sur le terrain ou dans le laboratoire, et au lieu de mettre en doute l'objectivité de toute observation du comportement, nous devrions aborder la difficulté d'une manière constructive et 
découvrir quels insights positifs, non susceptibles d'être obtenus par d'autres moyens, nous pouvons tirer du fait que la présence d'un observateur perturbe l'événement observé. » (1980 [1967], 369)

La question pertinente n'est dès lors pas de savoir si la présence d'une caméra perturbe ou non une situation - "I don't think it exists unaltered" dit Gregory Bateson dans une discussion sur les façons de filmer qui l'oppose à Margaret Mead (Brand, Bateson et Mead, 1976) -, mais de déterminer si la relation sociale engendrée par la pratique filmique induit une "modification significative" (Terrenoire, 1985, 519-520) et dans quelle mesure elle produit des données heuristiques concernant la situation d'observation susceptibles d'enrichir la connaissance des phénomènes étudiés. Cependant, même parmi les représentants de cette orientation, l'idée que la caméra puisse engendrer des perturbations spécifiques fait également débat.

7 Le terme "profilmique ", popularisé par les travaux de Claudine de France (1989 [1982]), est entré dans le langage courant de nombreux chercheurs en sciences humaines et sociales pour qualifier les effets engendrés par la présence d'une caméra sur l'activité qu'elle vise à décrire, effets qui reposeraient sur une forme de coopération plus ou moins vertueuse entre filmeur et filmé et se manifesteraient de façons diversement décelables ( $\mathrm{Cl}$. de France, 2006). Cependant cette notion ne fait pas l'unanimité. Aussi, selon Christian Lallier, la caméra n'aurait qu'une «incidence de forme " sur l'activité filmée : le dispositif filmique peut produire une "inflexion » et "renforcer les formes d'engagement des individus dans leur situation", sans pour autant créer "une situation inédite» $(2011,119)$. Tandis que certains parlent de profilmie, d'autres sont donc enclins à considérer que la présence d'un cinéaste n'a pas d'effets intrinsèquement différents de ceux engendrés par la présence d'un observateur a priori extérieur à une situation, faisant leur l'idée formulée par Jean-Dominique Lajoux selon laquelle «l'utilisation d'une caméra ne perturbe pas plus le milieu que l'ethnologue lui-même » $(1976,119)$.

8 Une façon de contribuer à alimenter la réflexion est de considérer le cinématographe dans sa totalité originelle : s'il est un outil d'enregistrement, il est aussi un outil de projection. Derrière son apparente banalité, une telle définition préalable est nécessaire car, de façon surprenante, les réflexions sur ce sujet semblent omettre de considérer que la situation filmique n'est qu'un premier maillon dans une chaîne de médiation audiovisuelle : si une caméra " prend des vues » et les fixe en images, cellesci sont elles-mêmes destinées à être visionnées. Avec la caméra, le cinéaste introduit non seulement un dispositif d'enregistrement par observation distanciée mais également un dispositif de visionnage par projection différée. Cette caractéristique qui fait toute l'utilité du film pour le chercheur (Cl. de France, 1989 [1982]) a pour particularité de placer, dès le tournage, l'acte filmé dans un espace-temps accessible à des personnes physiquement absentes de la situation filmée. Aussi faut-il considérer que la singularité de cet outil de prise/restitution de vues est de créer un dispositif de médiation faisant advenir un destinataire "virtuel $»^{4}$. Partant de ce constat, je défendrai ici l'hypothèse que si le dispositif filmique modifie effectivement quelque chose, c'est avant tout parce qu'il instaure une situation triadique filmé/filmant/ spectateur. Je suppose aussi que ce ne serait pas tant la présence physique du dispositif dans l'action en tant que tel qui serait susceptible de modifier une situation que la présence virtuelle de cet autre spectateur, la «troisième pointe du triangle » dont la caméra et le cinéaste sont "des représentants par anticipation » (Comolli, 2004, 163). Selon cette approche, le dispositif filmique est singularisé par la relation de 
transmission outillée qu'il instaure, la caméra opérant comme un médiateur entre la situation vécue et l'écran de projection.

9 Afin de mener cette réflexion autour des prétendus effets de la chaîne audiovisuelle, je me concentrerai sur l'étude des gestes en prenant appui sur une enquête ethnofilmique menée dans la ville de Mirecourt, le «berceau » de la lutherie française. Le présent texte est organisé en trois parties perméables qui proposent chacune de considérer le processus filmique selon des positions qui se déplacent du viseur à l'écran. Dans la première partie, il s'agira de se donner les moyens de penser le dispositif filmique dans le cadre plus large d'une réflexion sur les gestes qu'il convient ou non de montrer. Dans le cas de la lutherie, mais la remarque est valable pour d'autres situations, il faut se replacer dans le contexte plus général d'une profession où tout n'est pas fait pour être montré à n'importe qui. Ce constat préalable contribue à mieux comprendre pourquoi des gestes peuvent être filmés et d'autres non. Dans la deuxième partie, je propose de considérer le dernier temps de la chaîne de médiation, celui du visionnage des images et leur ambigüité. Il apparaît en effet particulièrement malaisé d'analyser nombre de techniques et de gestes présents dans les archives audiovisuelles. Seule une documentation précise sur les conditions du tournage et le contexte culturel de l'activité filmée permettent de mesurer sans naïveté les effets engendrés par le dispositif et de leur donner un sens. La dernière partie prendra appui sur un film décrivant le processus de fabrication d'un violon que j'ai réalisé avec un luthier enseignant à Mirecourt. Le dévoilement de la fabrique à quatre mains de ce film sera l'occasion d'enrichir la réflexion entamée dans les parties précédentes mais également d'interroger plus généralement l'éventuelle spécificité des gestes adressés à distance à « un autre » par le filtre de la médiation audiovisuelle.

10 Au-delà de l'ethnographie des luthiers qui sert à étayer la réflexion, ce texte invite à interroger la nature trouble du spectateur virtuel présent lors des opérations de prise de vues. En adoptant une telle optique, il apparaît possible de dépasser l'approche symptomatologique de la profilmie, considérant les effets (ou l'absence d'effets) occasionnés par la présence d'une caméra, pour tendre vers une approche que l'on pourrait qualifier d'étiologique, visant à considérer les raisons et les motivations (intentionnelles ou non) de ces éventuelles modifications. Appliquée à l'appréhension d'un flux gestuel par le film, la question pourrait alors être formulée ainsi : un geste filmé est-il un geste comme les autres et qu'est-ce que le film d'un geste nous dit de celui qui effectue ce geste?

\section{La cyane, l'aérographe et la télévision}

11 Certains luthiers m'ont laissé entendre que je ne pourrai pas filmer quelques-uns de leurs gestes, notamment ceux mobilisés lors d'opérations qu'ils ont mis des années à élaborer et ne souhaitent pas rendre accessibles au regard du premier confrère venu, préférant les transmettre à des collègues triés sur le volet. Il faut dire que les luthiers, particulièrement technophiles continuent à chercher en permanence des méthodes, des «trucs » qui permettront, l'espèrent-ils, de mieux faire sonner leurs instruments ou de leur conférer une bonne finition de surface. Certains de ces savoir-faire spécifiques ont été obtenus au terme de nombreuses expérimentations et l'on comprend aisément la réticence que peut ressentir un artisan à l'idée de les transmettre à n'importe qui. Il peut être utile de rappeler qu'autrefois certains maîtres 
demandaient aux apprentis de détourner le regard lorsqu'ils préparaient un mélange de vernis avant son application sur un coffre de violon en "blanc ». Si ce temps est sans aucun doute révolu, les luthiers d'aujourd'hui étant généralement disposés à transmettre l'intégralité de leur savoir - même si celui-ci ne se transvase évidemment pas d'un individu à l'autre comme un liquide entre deux récipients (Buob, 2012) -, il n'est pas pour autant question d'une totale transparence.

Il demeure donc des techniques qui ne sont pas bonnes à montrer car elles relèvent du domaine du secret. Mais ce n'est pas tant au cinéaste qu'il faut les cacher. Car le cinéaste, moi en l'occurrence, n'est pas lui-même luthier et il est incapable de voir ce qui fait la particularité des opérations effectuées. Certaines techniques peuvent donc être observées mais ne peuvent être filmées. Si elles ne peuvent l'être, c'est qu'un autre luthier, virtuel spectateur, risquerait de voir les images de ces gestes. C'est pour une raison comparable que récemment, alors que je filmais la fabrication de pâtes de fruits par des moines, le frère-confiseur m'a demandé de ne pas braquer ma caméra vers la recette écrite sur un cahier sans pour autant m'interdire de la lire directement, sans la filmer. Ici, la caméra matérialise un hypothétique concurrent auquel il faut cacher certains gestes de peur qu'il les reproduise.

Des gestes peuvent être cachés pour d'autres raisons. Dans l'univers de la lutherie, la question de l'apparition à l'image d'un simple accessoire technique, pourtant utilisé par tous, peut être très problématique. Tel est le cas de la colle cyanoacrylate, appelée simplement «cyane» par les luthiers, qui contient, outre ses vertus adhésives, une controverse sur ce qu'il convient ou non de montrer. Utilisée par chacun de nous comme colle forte, la cyane est aujourd'hui employée par la plupart des luthiers pour effectuer des opérations spécifiques (principalement pour coller, sur la touche de l'instrument, le sillet sur lequel viendront reposer les cordes). Toutefois nombre d'artisans considèrent qu'il n'est pas bon de la faire apparaître à l'image : symbole d'une modernité d'introduction récente, la cyane ne colle pas à l'image du luthier habituellement véhiculée.

Empreints d'une aura d'ancestralité, les luthiers ont vite appris à contrôler les apparences pour alimenter l'imaginaire d'une profession strictement artisanale. Un luthier m'a relaté qu'au XIXe siècle, Jean-Baptiste Vuillaume, le «Stradivarius français", participait de l'entretien d'une image de la lutherie comme travail exclusivement manuel dénué de toute intervention mécanique, perpétuant en cela l'idée que les méthodes des grands maîtres italiens des XVIIe et XVIIIe siècles étaient demeurées inchangées : les visiteurs de passage dans son atelier voyaient le vernis être appliqué au pinceau sur les instruments alors que dans les coulisses ils l'étaient à l'aérographe. Si cette assertion est historiquement douteuse (le brevet de l'aérographe a été déposé en 1888 et Jean-Baptiste Vuillaume est décédé en 1875) et à ma connaissance ne se fonde pas sur des faits avérés, elle traduit cependant l'expression de la mise en avant d'une identité de façade ${ }^{5}$ encore, par certains aspects, pertinente - il n'est point question ici de chercher à dénoncer une pratique répandue et banale: l'image qu'un artisan accepte de donner à voir de sa profession ne correspond pas toujours à la réalité de sa pratique quotidienne. Les termes de cet aménagement ne sont pas univoques, puisque la composition de cette façade est le fruit d'une coconstruction répondant en même temps aux attentes, véritables ou supposées, de musiciens en quête de l'image d'une profession ancestrale. Une anecdote qui m'a été relatée par un autre luthier est à ce titre très éclairante. Selon ses dires, il possédait 
dans sa boutique un instrument récemment terminé qu'il avait doté d'un vernis «neuf». Un musicien venu l'essayer ne fut pas du tout convaincu de sa sonorité le trouvant trop « vert ». Le luthier l'invita alors à revenir quelques semaines plus tard, devant entre temps, lui dit-il, recevoir alors en dépôt-vente des instruments qui pourraient lui plaire. Une fois le musicien parti, le luthier retira le vernis de l'instrument et entreprit de le vernir de nouveau en le patinant de façon à lui donner un aspect ancien. Lorsque le musicien revint pour l'essayer, il fut cette fois séduit par l'instrument le trouvant beaucoup plus « rond » que celui qu'il avait essayé quelques semaines auparavant.

Le vernis est à l'instrument ce que la façade est à l'atelier : il se doit généralement de s'inscrire dans une histoire longue précédent les révolutions industrielle et numérique. Aujourd'hui encore, des luthiers installent leurs machines électriques ou numériques dans des salles à l'abri des regards des visiteurs, préférant mettre en avant le versant manuel de leur activité. Dans la lutherie, et il en va de même dans de nombreuses activités artisanales, est souvent masqué ce qui risque de donner une image trop moderne de l'activité. Cependant si ces différentes anecdotes m'ont été relatées, c'est surtout afin de dénoncer une certaine tendance de la profession à survaloriser une approche passéiste. Car parallèlement des luthiers se refusent à cacher leurs machines (le recours à des méthodes modernes pouvant même être mis en avant) et des musiciens, revenus de l'idée que les instruments modernes ne pourraient pas être en adéquation avec leur quête acoustique, accordent moins d'importance à l'apparence de façade. Cacher la cyane ou au contraire accepter qu'elle soit filmée témoignent alors de deux modes d'engagement singuliers des personnes filmées dans l'entreprise cinématographique. Par le premier, le luthier vise à valoriser une image traditionnelle et «authentique» de la profession, tandis que par le second, le luthier accepte de donner à voir la réalité technique de son activité. Par le biais de cet engagement public, le luthier prend position vis-à-vis de ses confrères en même temps qu'il s'adresse aux musiciens. Aussi, accepter d'être filmé en train d'utiliser de la cyane est un acte idéologique fort, l'expression d'un point de vue sur la profession contenu dans un geste en apparence banal.

Indépendamment de la réflexion propre à la lutherie et à un niveau plus général, les différentes remarques qui précèdent permettent de considérer les gestes techniques selon deux grandes catégories perméables : d'une main, ceux qui sont destinés à être montrés, de l'autre, ceux qu'il conviendrait de masquer à certains yeux et en conséquence, par anticipation, à ceux du cinéaste. Le geste relève non seulement du faire mais également du montrer, surtout lorsque s'immisce au cours de l'exécution technique un destinataire autre que le matériau en cours de transformation. Ce postulat est essentiel dans le cadre de la réflexion qui nous intéresse ici, car si l'on peut admettre que dans le cinéma documentaire le caméraman est généralement amené à filmer des situations qui ne sont pas prévues pour l'être (Lallier, 2011, 118), il faut également convenir que dans les activités à dominante matérielle nombre de gestes peuvent - voire, sont faits pour - être observés et sont donc adressés (Buob, 2013b). Dans ce cas, la caméra vient renforcer un dispositif de médiation ou matérialiser un tel dispositif existant à l'état virtuel. La caméra fait ici advenir une situation de transmission qui lui préexiste, et le profilmique, s'il faut ainsi le qualifier, actualise alors une configuration de transmission potentielle. En faisant basculer le geste dans un schéma de transmission, en offrant la possibilité d'une télévision ${ }^{6}$ différée du geste, la présence de la caméra fait advenir un spectateur trouble, un hybride du confrère et 
du client - mais aussi de l'apprenti, de l'esthète ou du béotien comme on le verra ciaprès -, et renforce la dimension symbolique d'un geste sinon dominé par une visée principalement opératoire.

\section{Des voûtes, une tête en l'air, un plateau et les destinataires du geste}

17 Il est courant de considérer que les informations présentes dans une image sont inépuisables, qu'il est toujours possible d'y déceler des détails, d'en inférer des données utiles au chercheur. Dès lors qu'il a été filmé, en tant que document objectif du passé, un geste deviendrait une archive pouvant faire l'objet de recherches spécifiques susceptibles d'enrichir profondément la connaissance: "Un film de cinq minutes tourné dans l'Athènes du Vème siècle modifierait de fond en comble la vision que nous en donnent les historiens » soutient Claude Lévi-Strauss (Eribon, 1988, 169-170). Cette idée repose sur le postulat spéculaire que le geste filmé est un décalque de l'activité habituellement exercée. Or, comme cela vient d'être suggéré, il faut considérer que le dispositif filmique peut, dans certains cas, engendrer d'importantes modifications et que le geste filmé peut se révéler, en conséquence, particulièrement malaisé à analyser. L'étude de plusieurs archives filmiques de luthiers au travail me servira à poser les termes de cette aporie.

Dans une première archive, la caméra se déplace au sein des locaux de l'usine Laberte de Mirecourt (Guibert, 1966), une des grandes sociétés où jusque dans les années 1970, hors de l'«atelier des artistes", certains luthiers spécialisés travaillaient à l'effectuation d'opérations parcellisées en vue de la fabrication de violons. La caméra se rapproche d'un luthier effectuant des opérations de «façonnage de voûte sur coffre ». Une autre archive présente le luthier Paul Hilaire à son établi avec, entre ses mains, un moule dit " extérieur» (Davy, 1971). Les luthiers ayant eu l'occasion de visionner ces deux films ont alors dû reconsidérer certaines certitudes quant à l'histoire des techniques locales. Il est généralement admis parmi les luthiers que dans la «méthode Mirecourt ", les artisans, préférant finir les voûtes avant leur assemblage et la constitution du coffre, ne pratiquaient pas la technique dite de façonnage de voûte sur coffre, considérée comme le propre de la méthode italienne (Buob, 2013b). La seconde archive montre un des derniers luthiers de Mirecourt durant les années 1970 avec entre les mains un moule qui ne correspond pas à ceux habituellement associés à la méthode locale, alors que ce luthier est lui-même reconnu comme étant un de ses représentants typiques; on considère habituellement que la lutherie française a usé presque exclusivement de moules dits « intérieurs".

Les archives sont considérées comme des " preuves » venant contredire des certitudes. Cette remise en question repose sur l'idée que la présence de la caméra n'a pas modifié les façons de faire des luthiers filmés. Il peut en effet être supposé sans trop de risque, au vu de la maîtrise des gestes effectués, que le luthier de l'usine Laberte, pleinement engagé dans son activité répétitive, procède comme il a l'habitude de le faire; comme Claudine de France l'a justement spécifié, «l'effet profilmique est d'autant moins marqué que l'être filmé est étroitement soumis aux contraintes de la matière au sein d'une coopération immédiate, ou pris dans une relation fusionnelle avec son groupe d'appartenance " (Cl. de France, 2006, 129). Cependant la deuxième archive ne permet pas d'être aussi affirmatif. Si l'on voit effectivement un moule extérieur, le luthier n'est 
pas pour autant en train de l'utiliser, il est simplement posé sur l'établi, entre ses mains. A-t-il pour habitude d'utiliser ce type de moule? L'a-t-il posé sur l'établi pour montrer au cinéaste d'autres méthodes que celle qu'il utilise couramment? Tout au plus peut-on affirmer que ce genre de moule était présent dans l'atelier du luthier au moment où il a été filmé. Encore que le cinéaste a pu lui-même apporter l'objet pour questionner le luthier sur son usage. Quoiqu'il en soit, la présence de ce moule extérieur n'est pas aussi surprenante, car certains luthiers férus de l'histoire de leur profession savent que, contrairement à l'idée la plus répandue, ce type de méthode pouvait être utilisé dans les ateliers de Mirecourt.

Dans une autre archive datant de 1958, le luthier Lucien André Ragot, installé à Céret, effectue face à une caméra les étapes les plus caractéristiques de la fabrication d'un violon (Anonyme, 1958). Après avoir façonné le coffre d'un instrument, on le voit tailler la tête d'un violon. Sa façon de procéder peut surprendre les luthiers, car il effectue ce travail « en l'air ». C'est-à-dire qu'il ne pose pas la tête du violon sur l'établi, selon la méthode aujourd'hui présentée comme étant celle transmise à Mirecourt de générations en générations. Or cet artisan a appris le travail de son père qui lui même l'a appris d'un des grands maitres formateurs de Mirecourt: élève du luthier Léon Mougenot, le père de Lucien André Ragot a ensuite été employé chez Jacquet, Gand et Jacquemin, autant de luthiers parmi les plus fidèles à l'école française. Lucien André Ragot ne devrait-il pas en conséquence avoir acquis les gestes propres à la méthode de Mirecourt? Il est aujourd'hui impossible de savoir si cette méthode était celle effectivement employée par le luthier ou si elle l'a été de façon exceptionnelle pour le film. En effet, une multitude d'hypothèses peuvent être formulées : à Mirecourt, les têtes étaient généralement confectionnées par des artisans spécialisés (davantage menuisiers que luthiers), aussi, une fois installés hors de la ville, les luthiers formés à Mirecourt ont peut-être été contraints de développer des techniques singulières. Peutêtre que le procédé de fabrication des têtes n'était pas aussi standardisé que le discours habituel le laisse penser. Le cinéaste a peut-être demandé au luthier de travailler « en l'air » pour rendre les actions effectuées davantage accessibles à sa caméra (pour des raisons d'exposition et/ou de cadrage). Le luthier n'a peut être pas pris le temps d'installer les presses habituellement utilisées pour faire ce genre d'opérations étant donné qu'il ne s'agissait que de mettre quelques coups de gouge. Peut-être que le fait de sculpter une tête en l'air étant particulièrement ardu, l'artisan voulait-il faire montre de sa maîtrise. Faute de données accompagnant ce film et d'enquête minutieuse sur cet artisan, rien ne permet d'interpréter ce geste avec certitude. Sans les informations précises concernant aussi bien le hors-champ que le champ, les contraintes techniques et les intentions tant de l'artisan que du cinéaste, cette information technique de prime abord très riche perd tout intérêt scientifique.

Pour creuser davantage cette idée, considérons un autre exemple issu d'un travail effectué sur la dinanderie marocaine. Lors de cette enquête, j'ai été confronté à ce que j'ai proposé d'appeler, en empruntant le vocable de Claudine de France (2006), une macroprofilmie discrète et heuristique (Buob, 2009a, 368-369). Cette profilmie est macroscopique, car elle n'a pas concerné certains gestes mais le processus dans son ensemble. Elle est discrète, car un spectateur ne connaissant pas le processus n'en aurait pas conscience. Enfin, elle est heuristique, car elle a permis une présentation désencombrée facilitant l'appréhension du processus. 
Ce média ne peut être affiché ici. Veuillez vous reporter à l'édition en ligne http:// journals.openedition.org/itti/1055

Le jour du tournage du film Tifor (Buob, 2009b), du nom du grand plateau circulaire dont la fabrication lui sert de fil conducteur, le responsable de l'atelier a opéré des changements dans son comportement habituel : il a participé directement à la ciselure d'un plateau qu'il a extrait d'un ensemble de pièces, faisant en sorte que ce plateau soit décoré de façon continue alors que ces objets sont généralement transformés en séries, lesquelles s'enchâssent les unes aux autres et masquent en partie la continuité logique $\mathrm{du}$ processus de fabrication. Le fait de proposer un seul plateau à l'observation filmique témoigne de la représentation que la personne filmée se fait de la façon dont il convient de représenter sa propre activité : la profilmie ici sert de support d'expression à une conception de l'artisanat fondée sur la pièce unique dans un contexte pourtant dominé par le travail sériel et parcellisé. Témoignage de la frustration liée à la parcellisation des tâches et façon de mettre en avant sa propre activité, la caméra jouant alors davantage comme un éclairage mettant en lumière des artisans rendus anonymes par l'actuelle organisation du travail (Buob, 2009c), cette forme de profilmie est également adressée à un spectateur particulier. Sans explications, un spectateur serait naturellement amené à penser que les pièces sont travaillées une à une, alors que tel n'est pas le cas. Ici les données concernant le contexte de tournage et le savoir afilmique sur l'artisanat considéré permettent non seulement d'identifier les modifications engendrées par la caméra mais aussi d'en comprendre les raisons. À l'image du musicien pour le luthier, je symbolisais pour le dinandier l'étranger en quête d'une image de l'artisan marocain loin d'exercer une activité dominée par la quête de productivité.

Plus ou moins consciemment, la personne filmée peut accentuer ou masquer certains traits de son activité, car, comme le dit justement Christian Lallier, une personne aura tendance à accepter "d'être filmée selon l'image qu'elle estime donner de son implication dans la situation et non simplement pour l'image de la situation ellemême » $(2011,119)$. Mais il faut cependant ajouter une nuance à cette proposition : si la personne filmée peut faire abstraction de la présence de la caméra, elle fera plus difficilement abstraction du spectateur supposé se trouver au bout de la chaine et elle acceptera d'être filmée selon l'image qu'elle estime devoir donner à voir hors du tournage. Car, la singularité de la caméra est, rappelons-le, d'introduire un nouveau destinataire. Or ce destinataire invisible et indéfini peut contribuer à renforcer la part de ritualité diffuse présente dans tous les actes du quotidien ${ }^{7}$. Le spectateur virtuel de l'activité technique filmée s'apparente alors au destinataire invisible du rite, cet observateur "censé voir et entendre ce que l'agent du rite, son destinateur et présentateur lui offre en spectacle, ce qu'il met en scène plus ou moins consciemment à son intention », et qui « incarne les valeurs du groupe » (Cl. de France, 1993, 121).

Les différents exemples de changements induits par la présence de la caméra évoqués jusqu'à présent affectent principalement la façade des activités et l'agencement de ses composantes. Cependant le cas de Lucien André Ragot introduit l'idée que les changements dits profilmiques peuvent également se développer au niveau du geste lui-même. D'ailleurs comme le propose Claudine de France, l'« auto-mise en scène spontanée " engendrée par la présence du dispositif de médiation audiovisuel est le plus souvent d'ordre gestuel $(2006,120)$. Un autre film que j'ai réalisé en collaboration 
avec un luthier de Mirecourt offre un exemple de modifications qui affectent les mouvements de l'artisan et permet de mieux cerner les implications de la mise en visibilité de l'activité matérielle engendrée par le film.

\section{De l'établi à l'écran : des gestes professionnels aux gestes de métiers}

Le Musée de la lutherie et de l'archèterie de la ville de Mirecourt m'a passé commande d'un film sur le processus de fabrication d'un violon devant être diffusé dans le parcours de son exposition permanente (Buob, 2013c). J'ai alors demandé à un des luthiers enseignant au sein de l'école nationale de lutherie de Mirecourt de bien vouloir « jouer le jeu ». Afin de permettre une réalisation brève de ce film, nous avons décidé de mettre en place un dispositif de tournage particulier: la fabrication d'un violon nécessitant plusieurs semaines d'activité, nous avons pris le parti de ne pas en filmer l'intégralité mais les moments clés (il nous aura cependant fallu trois jours et demi de tournage). Pour ce faire, le luthier a réuni des pièces à différents stades d'achèvement ; par exemple : une table non creusée, une table terminée; des éclisses non pliée et une couronne d'éclisse terminée; un violon en blanc et un violon vernis. Grâce à la présence de ces différents éléments, il était possible de créer des ellipses pour réduire des étapes de travail longues et répétitives. Installé dans un atelier où personne n'était susceptible de nous déranger, nous avons fermé les volets afin de conserver une lumière ambiante constante permettant au moment du montage de masquer les éventuelles ellipses et le non respect de l'ordre des étapes filmées. Aussi ce film n'est pas un film sur la fabrication d'un violon mais un film sur différentes opérations permettant la fabrication des différentes parties d'un violon.

Ce média ne peut être affiché ici. Veuillez vous reporter à l'édition en ligne http:// journals.openedition.org/itti/1055

Si nous avons créé en concertation un dispositif pour réduire le temps de tournage, le luthier a de lui-même apporté des modifications dans les éléments de composition de son activité. Il a modifié certains éléments de façade de façon à inscrire ses actes dans un passé représentatif d'une image traditionnelle de la lutherie : utilisation d'un pot de colle en cuivre, d'un vieux fer à plier moins pratique mais plus ancien que l'habituel, d'un lacet pour fixer la touche au manche suivant une méthode aujourd'hui désuète, etc. Cependant la perspective du film a engendré d'autres changements, moins visibles. Le luthier a en effet porté une attention particulière à la façon d'effectuer chacun de ses gestes.

L'école française de lutherie revendique haut et fort la capacité de ses artisans à faire en deux coups de canif amples ce qu'un autre obtiendra maladroitement en une dizaine de petits gestes. Le geste se doit d'être à la fois direct et ample dans une recherche d'efficacité et de rentabilité (Buob et Klein, 2012 ; Buob, 2013b). Aussi le luthier filmé at-il donné de l'amplitude à ces gestes, une amplitude qui n'est pas aussi marquée lorsqu'il travaille pour lui. Le film réalisé pour le musée donne donc à voir la représentation en acte que le luthier filmé se fait de la façon dont il convient selon lui de représenter les gestes maîtrisés dans la lutherie française (Buob, 2013b). À travers ce film, le luthier ne se contente pas de profiter d'une forme de valorisation personnelle, il 
donne à voir ce qu'il veut que les apprentis, les musiciens et les béotiens voient, tout en se conformant à la perception que la majorité des membres d'une profession se fait de cette pratique dans le berceau de la lutherie française.

Le luthier avait pleinement conscience que le film que nous étions en train de réaliser constituerait, une fois dans le musée, une archive, un élément patrimonial donnant à voir la façon dont on fait un violon à Mirecourt. Aussi, était-il évident à ses yeux qu'il se devait de proposer à la caméra un processus opératoire caractéristique de l'école française. En tant que représentant d'une méthode spécifique, il s'engageait, en acceptant d'être filmé, à respecter une composition, un ordre et une articulation très codifiés des phases, des opérations et des gestes. On pourrait alors être tenté de considérer les actes du luthier comme étant pleinement contrôlés, stratégie d'un individu strictement calculant, induisant la disparition de toute poiesis - gestes improvisés dans la confrontation avec la matière - au profit d'une stricte praxis modélisation contrôlée de l'action. Pourtant, même dans un contexte a priori aussi contrôlé que celui de ce film sur la fabrication d'un violon, l'invention, la création, l'improvisation adviennent, et la relation négociée entre la technique et la matière continue à opérer : lors du creusement de la mortaise de tête, le luthier a exprimé avoir inventé un "truc », après avoir poussé son canif alors qu'il avait jusqu'alors tendance à le tirer.

31 La distinction entre "gestes professionnels » et " gestes de métier $»^{8}$ offre un cadre pertinent pour comprendre certains effets dus à la présence de la caméra. Dans un cas comme celui de la lutherie, les "gestes de métier " se déploient dans un contexte de transmission et véhiculent «les codes propres au métier »: «l'initiateur du geste se présente comme le membre d'une communauté de pratiques qui affiche ses savoir-faire et ses valeurs » (Jorro, 2006). En revanche, témoignant d'une " approche singulière et contextuelle », les gestes professionnels, qui «intègrent les gestes du métier en les mobilisant d'une façon particulière » et « se déploient en fonction d'une analyse précise et rapide de l'activité ", se développent hors du contexte de transmission, lorsque l'artisan est en prise directe et individuelle avec la matière. Les deux dimensions opératoire et symbolique, synthétisées dans chaque geste, dominent respectivement dans les gestes professionnels et dans les gestes de métier ; même s'il faut évidemment admettre, dans la continuité d'une longue tradition d'études sur le geste et les techniques, l'impossible démêlement de l'« entrelacs de l'action dans ses dimensions opératoires et symboliques" (Jorro, 1998). Outre qu'elle introduit l'idée d'une permanente variabilité et pluralité gestuelle, une telle distinction suggère que chaque artisan, travaillant et modélisant un référentiel préétabli, est susceptible de mobiliser de façon privilégiée un régime de gestes selon les contextes.

32 Les gestes professionnels dominent dans les actions directement orientées vers l'efficacité matérielle, l'obtention optimale d'un résultat. Ces gestes sont surtout accessibles par la caméra quand la personne filmée adhère pleinement à l'objectif de laisser saisir dans la transparence son activité quotidienne (par exemple le luthier acceptant de montrer qu'il utilise de la cyane), ou lorsque les contraintes sont telles que l'initiateur du geste se trouve dans l'incapacité de modifier son activité (tel l'artisan de l'usine Laberte effectuant une reprise de voûte sur coffre). Les gestes de métier se retrouvent davantage dans les situations de monstration technique, situations dans lesquelles il s'agit non seulement par les gestes d'être efficace, mais également de tenir un discours gestuel réflexif sur les actes eux-mêmes et donc de transmettre un 
ensemble de valeurs. Relèvent de cette catégorie non seulement les situations de médiation technique comme l'apprentissage mais aussi nombre de situations filmiques. Le film faisant basculer le geste dans un régime de monstration.

Si toute technique matérielle contient ce double registre par lequel la circonstance d'engagement de l'acteur relève non seulement de l'action proprement dite, mais aussi de la représentation de l'action, une technique matérielle est souvent destinée à être observée, s'inscrivant alors dans un schéma de transmission. La perspective d'une télévision relève de ce régime spécifique et l'acte en train de se faire peut alors s'accompagner d'une amplification de la dimension symbolique du geste au détriment de sa dimension opératoire. Mais il ne s'agit ici que d'une amplification car jamais une dimension ne peut totalement étouffer l'autre.

\section{Un geste, quatre mains, d'innombrables yeux}

34 L'idée d'une spécificité du geste filmé a été avancée en raison de la fonction médiatique de l'activité de tournage : accepter d'être filmé, c'est accepter l'idée que ce qui est filmé puisse être rendu public, vu par un tiers hors de l'espace-temps de l'action. Autrement dit, l'idée qu'il puisse exister des effets spécifiquement profilmiques sur les comportements tient à la singularité médiatique du dispositif: les activités filmées s'orientent certes vers les autres êtres matériels présents dans la situation (et le cinéaste en fait partie), mais s'adressent également par le truchement de la caméra à l'être immatériel qu'est l'observateur absent. Conçu comme dispositif de médiation, le dispositif filmique est cependant singulier en raison du statut hétérogène et trouble du spectateur. S'agit-il d'un béotien, d'un confrère, d'un apprenti, d'un musicien, etc. ?

Ne sachant pas clairement qui peut être amené à " revoir » ses gestes ou à écouter ses paroles, l'individu filmé est confronté à un "trouble de l'adresse » et « ne peut faire autrement que de s'adresser à lui-même " (Comolli, 2004, 164); Gérard Althabe développe la même idée lorsqu'il suggère qu'en raison du caractère "flou » du destinataire lors du tournage, l'entretien filmé « est un dialogue que l'on transforme en monologue " $(2001,41)$. En raison du retour réflexif sur les actes engendré par l'ambiguité de cette situation médiatique, les gestes ne sont alors plus uniquement dominés par la perspective d'un aboutissement, d'une transformation efficace de la matière, mais aussi par celle de la matérialisation d'un processus. Or, comme le soutient Anne Jorro, «lorsque le processus préoccupe l'initiateur du geste, le mouvement prolonge une intention, une manière d'être en relation avec le monde ", et l'initiateur du geste « développe alors une épistémologie et une éthique de l'action en réfléchissant à ses savoirs d'action » (1998). Si la présence d'une caméra peut avoir dans certains cas une simple incidence de forme par laquelle la personne filmée «renforce sa représentation de "personne absorbée par sa tâche" »(Lallier, 2011, 119), dans d'autres situations elle fait advenir un autre régime de pratique que celui qui prévaut généralement dans l'intimité de l'atelier.

Lorsqu'un geste est adressé, son initiateur se soucie de l'autre - en ce cas, «faire un geste c'est penser l'autre» (Jorro, 2006) - et le tournage d'un film a justement cette singularité de faire surgir cet autre, faisant ainsi basculer le geste du côté de l'adresse à un tiers. Le retour sur soi occasionné par la présence d'une caméra, contribue certes à amplifier le «travail d'objectivation que tout acteur effectue sur sa situation » (Lallier, 2009, 127). Mais, ce faisant, le regard réflexif, présent à un degré ou à un autre dans 
toute activité et amplifié par le dispositif filmique, projette les déploiements corporels dans une dimension langagière, une pratique adressée à un hypothétique spectateur. Traduisant un mode d'engagement différent de celui de l'artisan pleinement pris dans des actes de transformation, les gestes adressés et mis en image peuvent se lester d'une dimension symbolique accrue, devenus véhicules d'un discours spécifique.

Bien que fondamentale, la question du regard réflexif porté par l'individu sur ses propres actions est insuffisamment considérée par les recherches traitant de l'approche filmique des activités techniques. En effet celles-ci ont tendance à omettre de considérer que l'agent peut être envisagé comme le propre spectateur de ses actes et à minimiser l'impact des observateurs extérieurs, directs ou indirects, sur l'action. Pour Claudine de France, toute action est constituée d'un maillage de dimensions corporelles, matérielles et rituelles. Et le rite a pour fonction singulière de «s'offrir en spectacle » (1989 [1982], 71), non seulement aux observateurs visibles mais aussi aux entités invisibles. C'est justement ce qui, selon elle, permet de distinguer les activités rituelles des activités matérielles:

«[...] les techniques matérielles se distinguent des rites en ce que les activités concrètes, observables, qui les composent, ne se donnent pas en spectacle en tant que procès matériel de fabrication ou d'acquisition à un observateur, si ce n'est à leur propre agent de l'action. [...] En un mot, bien que les techniques matérielles se déploient parfois en fonction d'un spectateur immédiat (par exemple un inspecteur du travail) auquel il convient de montrer certaines choses et d'en cacher d'autres, leur véritable spectateur est celui du produit fini, non celui de l'activité qui est à l'origine de ce produit. » (Cl. de France, 1989 [1982], 72)

La figure de l'inspecteur du travail mentionnée par Claudine de France est proche de celle de chercheur observant que Christian Lallier utilise, en faisant référence à l'« Effet Hawthorne ", pour expliquer l'impact que la présence d'un observateur extérieur peut avoir sur le déroulement de l'activité gestuelle $(2009,29)$. De tels exemples laissent entendre que l'acte d'observation orienté vers les techniques matérielles serait une conséquence des formes modernes d'organisation du travail ou dus à la présence d'un dispositif de recherche et qu'en conséquence les gestes destinés à transformer la matière se dérouleraient majoritairement sans observateur ou alors de façon exceptionnelle - et c'est même ce qui les distinguerait du régime rituel. Ce postulat initial au nom duquel certaines activités ne sont pas destinées à être montrées, à s'offrir en spectacle, est présent chez les tenants comme les opposants de la notion de profilmie ${ }^{9}$. Pourtant, il faut admettre que dans de nombreux contextes techniques, les gestes sont observés et même que nombre de gestes sont faits pour être observés. À Mirecourt, rares sont les gestes qui ne sont pas observables : la plupart des luthiers reçoivent des visites régulières d'amis, de clients, de collègues et d'apprentis. Certes les luthiers passent beaucoup de temps seuls en prise directe avec le matériau, mais il ne s'agit là que d'un cas de figure parmi la diversité des régimes de gestes, car les gestes peuvent relever aussi bien du domaine du faire que de l'apprendre, du jouer ou du montrer, comme le souligne avec justesse François Sigaut (2010 [2002]); encore faut-il ajouter que ces registres se superposent dans la pratique et que les activités relevant exclusivement du faire effectuées en solitaire échapperont toujours à l'observateur. La caméra, en introduisant un dispositif de médiation, donne donc à voir les gestes en les offrant en spectacle. Si elle peut mettre en visibilité des gestes qui habituellement ne le sont pas, il ne faudrait pas pour autant en faire une généralité, car, bien souvent, le dispositif filmique actualise une situation de monstration potentielle. 


\section{Profilmie ou transfilmie?} «transfilmiques» ne sont que des cas particuliers parmi l'ensemble des effets "protélévisuels» créant les conditions d'une "mise en public ", c'est-à-dire des comportements plus ou moins conscients effectués en vue de leur appréhension visuelle différée par un tiers. Autrement dit le dispositif filmique n'est qu'un exemple parmi ceux susceptibles d'induire des comportements spécifiques en faisant advenir, par une publicisation, la possibilité d'une vision à distance de ses propres actes par un destinataire aux contours troubles. Une telle prise de conscience est fondamentale non seulement pour le chercheur préoccupé par l'étude de gestuelles, car bien souvent il ne travaille pas tant à partir de gestes qu'à partir d'images de gestes, mais plus généralement pour tout chercheur préoccupé par l'analyse des images documentaires et des conditions de leur obtention. 


\section{BIBLIOGRAPHIE}

Althabe G. (2001), « Lecture ethnologique du film documentaire », L'Homme et la société, n 142, p. 9-25.

Anonyme (1958), Fabrication de violons, Office national de radiodiffusion télévision française, Marseille, 6 minutes [notice INA : RAF04029970].

Brand S., Bateson G. et Mead M. (1976), "For God's sake Margaret”, CoEvolution Quarterly, n 10, p. 32-44.

Bril B. (2012 [1984]), « Description du geste technique : Quelles méthodes ?», Techniques \& Culture, $\mathrm{n}^{\circ}$ 54-55, p. 243-244.

Boukala M. (2009), Le dispositif cinématographique. Un processus pour [re]penser l'anthropologie, Paris, Téraèdre.

Buob B. et Klein V. (dir.), 2012, Luthiers, de la main à la main, Arles, Actes-Sud.

Buob B. (2013a), Luthiers de la main à la main, Musée de la lutherie et de l'archèterie française/ Palaviré productions/Vosges télévision, 80 minutes, [en ligne], URL : https://vimeo.com/ 95504460

Buob B. (2013b), « De l'adresse. Remarques sur les gestes du luthier », Gradhiva, no 17, p. 70-91. Buob B. (2013c), « Fabrication d'un violon », Luthiers de la main à la main, Musée de la lutherie et de l'archèterie française-Palaviré productions, 32 minutes, [en ligne], URL : http://vimeo.com/ 22691598

Buob B. (2012), « Du vol à l'appropriation du savoir. Controverse sur l'apprentissage à Mirecourt ", Luthiers, de la main à la main, Arles, Actes-Sud, p. 136-151.

Buob B. (2009a), La dinanderie de Fès, un artisanat traditionnel dans les temps modernes. Une anthropologie des techniques par le film et le texte, Paris, Ibis Press-Éditions de la MSH.

Buob B. (2009b), « Tifor », La dinanderie de Fès, un artisanat traditionnel dans les temps modernes. Une anthropologie des techniques par le film et le texte, Paris, Ibis Press-Éditions de la MSH, 70 minutes, [en ligne], URL : http://vimeo.com/76049234

Buob B. (2009c), « Filmer, entendre, montrer des savoir-faire. Regards et écoutes croisés dans la médina de Fès ", Ateliers d'anthropologie [En ligne], no 33, mis en ligne le 18 mars 2009, consulté le 01 novembre 2016. URL : http://ateliers.revues.org/8206

Buob B. (2004), «Ethnovidéographie des dinandiers de Fès : approche d'un terrain d'ethnologie avec la vidéo numérique », Actes des premières rencontres d'anthropologie du Maghreb, Rabat, Centre Jacques Berque, p. 71-93.

Comolli J.-L. (2004), Voir et pouvoir. L'innocence perdue : cinéma, télévision, fiction, documentaire, Lagrasse, Verdier.

Daston L. et Galison D. (2012 [2007]), Objectivité, Bruxelles, Les presses du réel.

Davy F. (1971), A propos du congrès national des luthiers, Office national de radiodiffusion télévision française Nancy, 4 minutes [notice INA : SXF01012677].

Devereux G. (1980 [1967]), De l'angoisse à la méthode dans les sciences du comportement, Paris, Flammarion. 
Eribon D. (1988), De près et de loin. Entretiens avec Claude Lévi-Strauss, Paris, Odile Jacob.

France Cl. de (2006), «La profilmie, une forme permanente d'artifice en documentaire », Travaux en anthropologie filmique, Nanterre, Publidix, p. 117-142.

France Cl. de (1993), « Le destinataire du rite et sa mise en scène filmique », Cinéma, rites et mythes contemporains, $\mathrm{n}^{\circ} 16, \mathrm{p} .121-143$.

France Cl. de (1989 [1982]), Cinéma et Anthropologie, Paris, Ed. de la MSH.

France X. de (1985), Réflexions sur le cinéma direct, Nanterre, Université Paris 10 (prépublications de la Formation de recherches cinématographiques), $\mathrm{n}^{\circ} 6$.

Guibert E. (1966), Les luthiers de Mirecourt, Office Radio Télévision France, 8 minutes [notice INA : SXF01015269]

Jorro A. (1998), «L’inscription des gestes professionnels dans l'action », En question, nº 19.

Jorro A. (2006), «L'agir professionnel de l'enseignant », conférence au séminaire de recherche du Centre de Recherche sur la Formation, Paris, CNAM.

Lajoux J.-D. (1976), « Le film ethnographique », Outils d'enquête et d'analyse anthropologiques, Paris, Librairie François Maspero, p. 105-131.

Lallier C. (2011), « L'observation filmante. Une catégorie de l'enquête ethnographique », L'homme, $\mathrm{n}^{\circ}$ 198-199, p. 105-130.

Lallier C. (2009), Pour une anthropologie filmée des interactions sociales, Paris, Archives contemporaines.

Laplantine F. (2007), « Penser en images », Ethnologie française, $n^{\circ}$ 37, p. 47-56.

Latour B. (2006a), Changer de société, refaire de la sociologie, Paris, La Découverte.

Latour B. (2015 [2006]), « Sur un livre d'Étienne Souriau : Les différents modes d'existence », Étienne Souriau. Une ontologie de l'instauration, Paris, Vrin, p. 17-53.

Rouch J. (1968), « Le film ethnographique », Ethnologie générale, Paris, Gallimard, p. 429-471.

Sigaut F. (2010 [2002]), « La formule de Mauss », Techniques \& Culture, $\mathrm{n}^{\circ}$ 54-55, p. 357-367.

Souriau E. (2009 [1943]), Les différents modes d'existence, Paris, PUF.

Souriau E. (1953), L'univers filmique, Paris, Flammarion.

Terrenoire J.-P. (1985), « Images et sciences sociales : l'objet et l'outil », Revue française de sociologie, $\mathrm{n}^{\circ}$ XXVI-3, p. 509-527.

\section{NOTES}

1. Cette affirmation vient confirmer une intuition ayant fait l'objet d'une réflexion antérieure (Buob, 2013b).

2. J'ai détaillé ailleurs ce genre d'approche (Buob, 2009a, 359-397).

3. Suivant une posture qui se généralise en anthropologie audiovisuelle (Buob, 2004 ; Lallier, 2009 ; Boukala, 2009).

4. Le terme virtuel n'est pas à entendre dans le sens commun qui, l'associant aux technologies numériques, le range du côté de l'immatériel, l'oppose au réel. Le virtuel est ce qui existe en puissance et agit en retour sur l'actuel. 
5. Entendue dans un sens large d'inspiration goffmanienne, la « façade » désigne l'identité visible promue par le commerçant/artisan. Elle comprend aussi bien la vitrine, le décor, l'agencement de l'espace d'une boutique/atelier que les modes vestimentaires ou les gestes visibles, etc.

6. Le terme "télé-vision" doit ici être entendu dans son sens étymologique de "vision à distance ». Cette acception du terme a été récemment réintroduite par Damien Mottier dans son travail sur les usages des outils télévisuels comme technique de médiatisation de l'expérience religieuse par les mouvements pentecôtistes.

7. Empruntée à Claudine de France, la notion de ritualité diffuse exprime la prégnance des " règles » socio-culturelles qui toujours dans les activités quotidiennes, et notamment dans le domaine des techniques matérielles, s'entremêlent aux contraintes d'ordre physique: «Imprégnant en permanence chaque chose, le rite se confond, dans l'espace et dans le temps, avec les gestes indispensables à l'exécution du programme matériel le plus banal [...]. » (1989 [1982], 23)

8. Je reprends ici, tout en l'adaptant, la distinction proposée par Anne Jorro dans le contexte de recherche sur les pratiques enseignantes (2006).

9. Claudine de France est cependant plus nuancée que Christian Lallier qui oppose de façon à mon sens trop étanche les situations effectives aux situations fictives $(2009,227-233)$.

\section{RÉSUMÉS}

Un geste filmé est-il un geste comme un autre? Quels peuvent être les effets engendrés par la présence d'une caméra sur une technique matérielle et comment en tirer des vertus heuristiques? Ce texte propose d'appliquer ces questionnements, récurrents en anthropologie audiovisuelle, à certains gestes de fabrication de violons filmés lors d'une enquête sur l'apprentissage du métier de luthier. En concevant le cinéma comme un dispositif de médiation, l'hypothèse ici défendue est que la présence de la caméra fait advenir un spectateur virtuel et trouble dans la situation filmée qui incite à accroître le regard réflexif que l'agent porte sur ses propres gestes et, en conséquence, renforce la part de ritualité diffuse présente en chaque activité matérielle. Autrement dit, la caméra, en faisant advenir un observateur virtuel, comparable à certains égards au destinataire invisible d'un rituel, fait pencher le geste du côté de l'adresse à un tiers et accentue sa part d'expressivité au détriment de sa vocation opératoire.

Is a gesture, when filmed, still just like any other? What impact might the presence of a camera have during a technical activity and, from a heuristic perspective, what can it help us learn? This paper will explore such questions --regularly discussed in audiovisual anthropology--, by paying attention to some specific gestures filmed during an investigation about French violin makers. By considering movie-making as a mediation process, we intend to show that the camera's presence brings about the virtual occurrence of a rather blurry spectator of the sequence being filmed, thereby increasing the actor's reflexive stance on his or her own gestures. This, in turn, tends to reinforce the ritual aspects lingering behind each and every physical activity. In other words, by bringing about the presence of a virtual observer (in some ways comparable to the invisible recipient of any ritual), the camera tends to increase the gesture's expressiveness at the expense of its functional dimension. 
INDEX

Keywords : filmic ethnography, violin making, profilmic, reflexivity, potential spectator Mots-clés : ethnographie filmée, lutherie, profilmie, réflexivité, spectateur virtuel

\section{AUTEUR}

\section{BAPTISTE BUOB}

Formé à la fois à l'anthropologie et au cinéma, Baptiste Buob est chargé de recherche au Laboratoire d'ethnologie et de sociologie comparative (CNRS/Université Paris Nanterre). S'intéressant aux articulations entre pratiques audiovisuelles et ethnographiques, ses recherches relèvent plus généralement de l'anthropologie des techniques et de l'anthropologie filmique. 